\title{
Graft-Free Sinus Augmentation Procedure: a Literature Review
}

\author{
Ginnady Pinchasov ${ }^{1}$, Gintaras Juodzbalys ${ }^{1}$ \\ ${ }^{1}$ Department of Oral and Maxillofacial Surgery, Kaunas University of Medicine, Lithuania.
}

\author{
Corresponding Author: \\ Ginnady Pinchasov \\ Siaures 103-21, LT-49238, Kaunas \\ Lithuania \\ Phone: +37064333569 \\ E-mail: gabriel.genady@gmail.com
}

\begin{abstract}
Objectives: The restoration of edentulous posterior maxilla with dental implants is challenging due to a deficient posterior alveolar ridge. Over the last decade an advance in the graftless bone augmentation procedures had occurred where the space left beneath the Schneiderian membrane is filled with blood clot in order to produce bone formation. The aim of present article is to review the scientific literature with respect to bone formation in the sinus, after membrane elevation procedure, without using any bone substitutes.

Material and Methods: A comprehensive review of the current literature was conducted according to the PRISMA guidelines by accessing the NCBI PubMed database. The articles were searched from 1993 to 2013. English language articles with minimum one year patient follow-up and radiological and/or histological diagnostics of newly formed bone were included. Articles were excluded, if usage of bone graft or bone substitutes and/or usage of osteotome has been made during sinus lift operation.

Results: A total of 19 studies were included: 2 studies were related to the sinus membrane's osteogenic potential, 3 to the histological evidence of bone formation in monkeys and humans, 12 to the radiologic evidence of bone gain, and 2 to the space-maintaining management. $100 \%$ of the reviewed articles presented with increased bone formation and high implant survival rates resulting from the graft-free technique.

Conclusions: It is clearly shown in the review that the potential of the maxillary sinus to heal and to form new bone without bone grafts or substitutes is of high nature.
\end{abstract}

Keywords: bone formation; dental implants; maxillary sinus floor augmentation; Schneiderian membrane.

\author{
Accepted for publication: 8 March 2014 \\ To cite this article: \\ Pinchasov G, Juodzbalys G. Graft-Free Sinus Augmentation Procedure: a Literature Review. \\ URL: http://www.ejomr.org/JOMR/archives/2014/1/e1/v5n1e1ht.pdf \\ doi: $10.5037 /$ jomr.2014.5101
}




\section{INTRODUCTION}

The pyramid-shaped maxillary sinus (or antrum of Highmore) is the largest of the paranasal sinuses, and drains into the middle meatus of the nose. The sinus is lined with mucoperiosteum, with cilia that beat toward the ostia. This membrane is also referred to as the "Schneiderian Membrane", which is histologically a bilaminar membrane with ciliated columnar epithelial cells on the internal (or cavernous) side and periosteum on the osseous side. The size of the sinuses varies in different skulls, and even on the two sides of the same skull [1].

As a result of long term edentulism a decrease in the bone level of the alveolar process occurs due to postextractional bone resorption and sinus pneumatisation [2].

The aim of sinus lift procedure is to compensate this bone loss by creating increased bone volume in the maxillary sinus and thus enabling installation of dental implants. Initially, the most popular bone grafting materials are autogenous bone grafts and other bone substitutes [3]. The disadvantages of such methods are high costs for grafting material, time consuming and high morbidity, because harvesting of bone grafts is needed.

The results of a paper published by Troedhan et al. [4] discuss that the key role of the sinus membrane, as the main carrier of bone reformation after sinus lift procedures, as multiple experimental studies suggested. Thus the importance of minimal invasive and rupture free sinuslift procedures is underlined and does not depend on the type of grafting material used. The potential of new bone formation without bone graft in the maxillary sinus was presented in a variety of studies including radiological and histological evidence, and recent studies indicate high success rates of such method [6-10]. Because of the disadvantages of existing methods that was described above and because of the recent studies regarding the potential of new bone formation without bone graft, there is an importance in reviewing the existing information in the literature.

The aim of this paper is to review the scientific data regarding to the natural bone formation in the sinus, after membrane elevation procedure, without using any bone substitutes.

\section{MATERIAL AND METHODS Types of publication}

The review included studies on humans and animals published in the English language. Letters, editorials, $\mathrm{PhD}$ theses and abstracts were excluded.

\section{Types of studies}

The review included any published observational studies (cross-sectional surveys, cohort and casecontrol studies), clinical trials of treatment and case series. Single case reports were excluded.

\section{Population}

Studies of adult patients with performed graft-free sinus augmentation were selected.

\section{Graft-free sinus augmentation definition}

Graft-free sinus augmentation is a sinus lift procedure using the lateral window approach by Boyne [3] , while no bone graft or other substitute is being used for the bone gain beside blood clot.

\section{Outcome measures}

Studies with radiological and/or histological diagnostics of newly formed bone in the sinus were included.

\section{Literature search strategy}

According to the PRISMA guidelines [5] an electronic search was conducted using MEDLINE (PubMed) to locate articles concerning graft-free sinus lifts. The search terms used were: „sinus lift", „sinus membrane elevation“, „sinus augmentation“, „sinus lift without bone graft" and ,implant placement without bone graft". Due to the low number of relevant articles and to insure the sensitivity of the systemic review the articles were searched from 1993 to 2013. Bibliographies of the selected articles were also manually searched. Figure 1 illustrates the workflow of the comprehensive literature review. Titles derived from this broad search were independently screened by two authors based on the inclusion criteria. Disagreements were resolved by discussion. Full reports were obtained for all the studies that were deemed eligible for inclusion in this paper.

\section{Inclusion and exclusion criteria}

Inclusion criteria for the selection were:

- English language;

- Minimum one year patient follow-up;

- Radiological and/or histological diagnostics of 


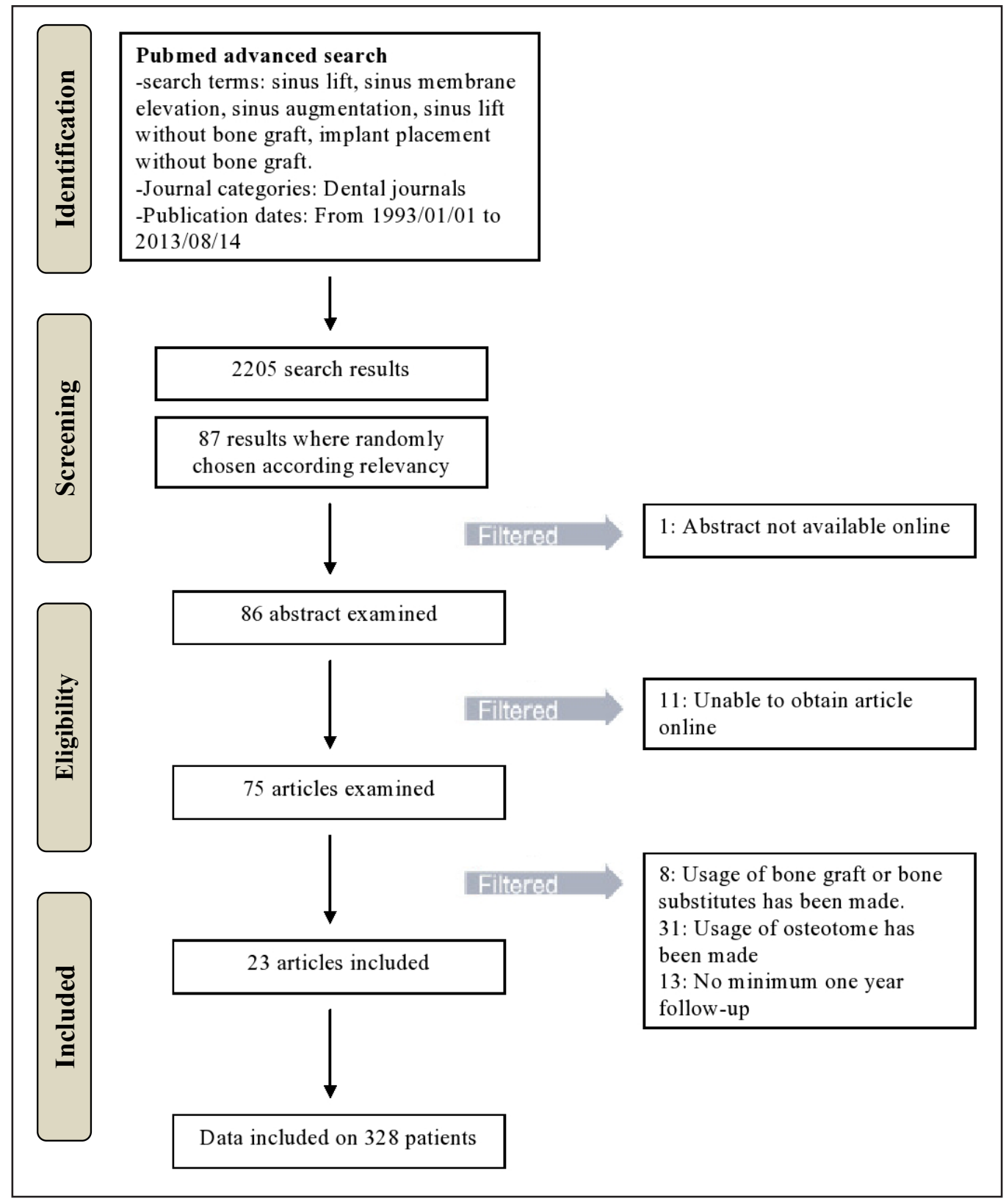

Figure 1. PRISMA flow diagram.

newly formed bone.

Exclusion criteria for the selection were:

- Usage of bone graft or bone substitutes has been made;

- Usage of osteotome has been made.

\section{Article review and data extraction}

The search delivered 2205 search results from which 86 abstracts were reviewed (Figure 1). A total of 75 articles were ultimately reviewed in full. Preliminary exclusion was made by the title and its relevancy, later by abstract and articles availability online. Finally, articles that did not meet the inclusion and exclusion criteria, where filtered as followed: usage of bone graft or bone substitutes has been made (8), usage of osteotome has been made (32) and no minimum one year follow-up was found (13). The data was included on 328 patients.

\section{Quality assessment}

The Cochrane collaboration bias summary for potential bias was used to assess the quality of studies and identify papers with intrinsic flaws in method and design [ $[\underline{6}$.

\section{RESULTS}

A total of 19 studies were included in this review. 2 studies were related to sinus membrane's osteogenic 
potential, 3 studies were related to histological evidence of bone formation in monkeys and humans, 12 studies were related to the outcome, implant stability and radiologic evidence of bone gain after using the graft-free augmentation, 2 studies were related to space-maintaining management (Table 1).

\section{Scheidrian membrane's osteogenic potential}

Studies, reviewed in this paper presented well a creation of new bone formation in the maxillary sinus by mucosal membrane lifting without the use of any graft material. Nevertheless, the mechanism of this specific bone gain is yet unknown. However, Srouji et al. [7], using both in vitro and in vivo assays, attempted to explore the bone formation beneath the sinus membrane on the maxillary sinus floor. The aim was to test the osteogenic potential of human maxillary sinus Schneiderian membrane (hMSSM). Samples of hMSSM were cultured and studied in histological manner. Flow cytometry analysis was conducted on cultures by adopting mesenchymal progenitor cell markers. Using relevant in vitro assays the potential to osteogenic differentiation by the hMSSM cells was analyzed. It was seen that the cells are capable of inducing and reviling different osteogenic markers such as osteonectin, osteopontin, osteocalcin, alkaline phosphatase, bone morphogenic protein-2, as well as to induce their extracellular matrix mineralization. Further, the osteogenic potential of hMSSM-derived cells was confirmed in vivo experiments, where cultured cells and a ceramic mix consisted from HA/ $\beta$-TCP were connected into a fibrin clot and implanted under the skin of thymic mice. After 8 weeks of healing formation of histology-proven bone at ectopic sites subsequent to transplantation of hMSSM-derived cells where present. It was deducted that genuine osteogenic potential is connected with the hMSSM and may be of great contribution to the development of successful sinus augmentation techniques. In 2010, Srouji et al. [8] tests whether such osteogenic activity relies on inherent osteogenic capacity, that existing in the sinus membrane using simulation in vivo clinical condition of sinus lifting in an animal model. Human Schneiderian membrane was folded around a fibrin clot that was later transplanted subcutaneously into mice. New bone formation was observed, as well as in the previous experiment, illustrating the innate osteogenic potential within the maxillary Schneiderian sinus membrane and its importance of understanding and potentially improving bone regeneration in sinus

Table 1. Description of studies included in the review

\begin{tabular}{|c|c|c|c|}
\hline Study & Year of publication & Species & Diagnostic evidence \\
\hline \multicolumn{4}{|c|}{ Scheidrian membrane's osteogenic potential (in vitro and in vivo) } \\
\hline Srouji et al. []] & 2009 & mice & histologic \\
\hline Srouji et al. [] & 2010 & mice & histologic \\
\hline \multicolumn{4}{|c|}{ Studies regarding implant placement after graft-free sinus lift } \\
\hline Palma et al. [9] & 2006 & Capuchin primates & histologic \\
\hline Boyne et al. [10] & 1993 & monkeys & radiographic \\
\hline Sohn et al. [11] & 2010 & humans & radiographic + histologic \\
\hline Lundgren et al. [12] & 2003 & humans & radiographic \\
\hline Lundgren et al. [13] & 2004 & humans & radiographic \\
\hline Sohn et al. [14] & 2010 & humans & radiographic \\
\hline Chen et al. [15] & 2007 & humans & radiographic \\
\hline Hatano et al. [ㅌ] & 2007 & humans & radiographic \\
\hline Moon et al. [17] & 2011 & humans & radiographic + histologic \\
\hline Thor et al. [18] & 2007 & humans & radiographic \\
\hline Balleri et al. [19] & 2010 & humans & radiographic \\
\hline Lin et al. [20] & 2011 & humans & radiographic \\
\hline Cricchio et al. [21] & 2011 & humans & radiographic \\
\hline Ellegaard et al. [22] & 1997 & humans & radiographic \\
\hline Ellegaard et al. [르] & 2006 & humans & radiographic \\
\hline \multicolumn{4}{|c|}{ Space-maintaining management with graft-free sinus lift } \\
\hline Johansson et al. [2ㄴ] & 2012 & humans & radiographic \\
\hline Kaneko et al. [를 & 2012 & humans & radiographic \\
\hline
\end{tabular}


lifting procedures in the future.

\section{Histological evidence of bone formation}

The first histological evidence to verify new bone formation was demonstrated in 2006 by Palma et al. [9] on four tufted capuchin primates that experienced maxillary sinus membrane elevation surgery using a replaceable bone window technique.

According to Palma et al. [9] study, no differences on new bone formation, implant stability and boneimplant contacts was demonstrated between two groups with and without adjunctive autogenous bone graft, furthermore stronger bone tissue response is seen in Oxidized implants than the control turned surface.

An alike clinical studies with monkeys was already described in 1993 by Boyne [10] where maxillary sinus floor augmentation on five monkeys was preformed, by simply elevating the maxillary sinus membrane without the use of bone grafting. Implants were placed bilaterally in the sinus perforating into it $5 \mathrm{~mm}$. Protruding implant in the right antrum was placed with bone graft and in the contra-lateral implant no bone grafts where used. Radiologic evidence of new bone was seen around the implant in which no bone graft was administered, though unlike Palma's et al. [9] study no histological evidence was yet to be shown.

Only 2 years afterwards a first histological evidence of new bone formation in the maxillary sinus with membrane elevation only was obtained on humans by Sohn et al. [11] where 21 implants were placed, with an average residual bone height of $5 \mathrm{~mm}$ after 6 months they where evaluated. All implants remained stable during the study period in clinical evaluations. Bone formation and new sinus floors were found in both radiographic and histological evaluations.

\section{Graft-free sinus procedure using lateral window approach}

Lundgren et al. [12] presented a case study in 2003 on a patient that needed a removal of mucosal cyst of the maxillary sinus and augmentation of the maxillary sinus in order to pace an implant later on. An intra-sinus mucosal cyst was removed through a prepared bony window in the lateral antral wall. The ruptured mucosa was sutured and the bone window was replaced. A space created by bony walls and sinus membrane had been formed. After 3 months of healing, clear radiographic signs of bone formation were observed. The space between the replaced bony window and the lifted sinus membrane was filled with newly formed bone. This was an unexpected healing pattern witch lead him to conclude that the creation of a space between the bone surfaces and the sinus mucosa in the combination of the surgical trauma result in spontaneous bone formation in the maxillary sinus. Since the predictability of the technique presented above is yet unknown in 2004 Lundgren et al. [13] conducted a study in order to investigate the possibility to perform sinus membrane elevation and facilitate implant placement without any additional bone grafting material and whether it is a valid technique.

The study group consisted out of 10 patients in whom a total of 12 maxillary sinus floor augmentations were performed and 19 implants were installed. The bony window was dissected, elevated superiorly, and sutured to the sinus wall to create and maintain a compartment for blood clot formation. The implants were installed then the bony window was replaced, and the flap was sutured into position.

During the study period all implants showed stability and clear signs of bone formation, this clearly demonstrated great potential of bone formation and regeneration capability in the maxillary sinus without the use of additional bone grafts. Lundgren et al. [13] concluded that in general it is achieved by the principle of guided tissue regeneration, but the precise mechanisms of this healing pattern are yet unknown.

As a result of previous studies that showed bone formation capability without bone grafts, in 2010 Sohn et al. [14] presented a study that demonstrating new bone formation in the maxillary sinus using absorbable gelatin only. The study consisted of seven patients treated with sinus augmentation with restorable gelatin sponge membrane and simultaneous implant placement. The lateral bony window was formed and the sinus membrane was elevated to make space. 18 implants were placed and the absorbable gelatin sponge was inserted in the new compartment under the elevated sinus membrane.

After an average of 6 months given for the implants to integrate, new bone formation in the maxillary sinus was seen on radiographs. It was mentioned that due to failed osseointegration 2 implants were removed. The study showed clearly that implant placement with gelatin sponge can be a predictable procedure for sinus augmentation without using bone graft.

In 2007, a study was published by Chen et al. [15] where maxillary sinus augmentation without bone graft using only blood was preformed in 33 patients. The average age of the patients was 55 years old. 47 implants were followed for more than 2 years after prosthesis delivery. The sinus mucosal membrane was elevated with the bony window still attached to 
the membrane (trap-door, open-window method). The implants were placed immediately with sinus lifting procedure. Patients where evaluated after 2 years displaying $100 \%$ of fixture survival.

Increases in bone height ranged from $3 \mathrm{~mm}$ to $9 \mathrm{~mm}$ with an average of $4.5 \mathrm{~mm}$ bone gain thus it was concluded that the survival rate was good.

In 2007, a case series was presented by Hatano et al. [16] of 6 patients that undergone a maxillary sinus floor augmentation using peripheral venous blood alone. After the elevation of a mucoperiosteal flap the lateral wall of sinus cavity was exposed. The piezoelectric saw with thin blade connected to piezoelectric device was used with copious saline irrigation to create the lateral window of the maxillary sinus. The bony window was detached carefully to expose the sinus membrane. The sinus membrane was carefully elevated from the sinus floor walls with a manual elevator. 14 implants where installed penetrating into the sinus cavity. The sinus cavity was then filled with patient's own peripheral venous blood. The bone window was replaced with medical tissue glue for closure of the potential gap in the bony window of the osteotomy thus preventing blood leakage from the created compartment in the maxillary sinus. After 6 months, the implants were evaluated. One of the 14 implants failed but successful new bone formation was found in all sinuses as seen from radiographs.

In 2011, Moon et al. [17] published a similar study to the one presented above by Hatano et al. [16] Moon's et al. [17] study results presenting with similar conclusions thus giving further justification and strengthens Hatano's et al. [16] study results. In the study, fourteen patients were consecutively treated and in total 17 sinus lifts had been made.

In Hatano's et al. [16] study lateral window approach was used. The lateral bony window was created using a piezoelectric saw, and the sinus membrane was elevated to make space. The sinus cavity was filled with venous blood and resorbable blast mediasurfaced dental implants were placed simultaneously. After 6.8 months, the sinus augmentations were evaluated and new bone formation in the maxillary sinus was seen by both radiographic and histological evaluation. Overal, implant survival rate was $93.5 \%$ and according to the histomorphometric data vital bone formation was $38.7 \%$. Of the 31 implants placed, 2 failed. It was mentioned in the study that all failures occurred when implants were placed into the extraction socket. It was established that a maxillary sinus floor augmentation using peripheral venous blood and simultaneous placement of dental implants is a reliable procedure and this is in coincidence with
Hatano et al. [16].

In 2007, Thor [18] presented a study, where 20 patients were treated with implant therapy. 44 Astra Tech implants, in total, were placed in the maxillary sinus (27 sinuses were lifted). Implants were followed every year in the period of time between November 2001 to June 2004 and all the implant installations carried without graft material. The sinus membrane elevation preformed in a matter where a bony window was removed from the maxillary anterior sinus wall. Implants were placed in the residual bone right after elevation of the mucosal membrane. During the surgery, perforations of the mucosal membrane was recorded together with the bone height. Abutments were connected after 6 months of healing. Evaluation after an average time of 27.5 months resulted in $97.7 \%$ of implants survival rate and $6.51 \mathrm{~mm}$ was the average gain of bone at the maxillary sinus. Although bone formation was successful few complications had occurred, in $41 \%$ of operated sinuses observed perforations of the maxillary sinus mucosal membrane and one implant was lost. Marked bone gain was observed around long implants as well as in the case when the residual bone below the sinus was low.

In 2010, Balleri et al. [19] presented a study, where fifteen patients were recruited for sinus augmentation in combination with implant placement without biomaterials. A mean residual bone height of the patients was $6.2 \mathrm{~mm}$. A replaceable bone window was opened and the membrane was dissected from the sinus walls. 28 of Astra Tech implants were installed in the residual crest (the implants were keeping the membrane in the elevated position). After 1 year, the implants, where evaluated and the average bone gain was $5.5 \mathrm{~mm}$. All of the implants survived at the end of the follow-up. It was established that the bone gain was less than the average bone gain in membrane elevation which is $8.2 \mathrm{~mm}$. Bone regeneration was less at the distal aspect of the most posteriorplaced implant. The height of membrane lift was not correlated with the amount of regenerated bone. In other words, the length of the implants was not related to the amount of gained bone unlike in Thor's [18] study from 2007, where greater bone formation was observed around the long implants. Not only all of the 28 implants were stable during the first year of loading and that no additional costs for biomaterials were necessary, but also problems concerning bone harvesting as morbidity were avoided.

In 2011, Lin et al. [20] presented a 5-year follow-up of 80 implants in 44 patients. The implants weere placed immediately after the lateral trap-door window procedure to accomplish maxillary sinus elevation without bone grafting. Surgeries were carried out with 
either local or general anesthesia and prophylactic antibiotics were given. Full-thickness flaps were carefully elevated following a midcrestal incision. Extreme care was taken to radically elevate the sinus membrane from the trap-door window. The floor, lateral wall, and posterior wall of the sinus membrane were detached and pushed upward and medially to allow the placement of dental implants into the bone chamber. After five years, the survival rate was $100 \%$. Before the treatment, $3 \mathrm{~mm}$ of residual bone height was required for inclusion and on average residual bone height was $5.1 \mathrm{~mm}$. New bone formation in the sinus was confirmed and the average gained bone height after five years was $7.4 \mathrm{~mm}$.

Cricchio et al. [21] presented a study were a total of 84 patients underwent 96 membrane elevation procedures and simultaneous placement of 239 implants without adding any graft material. Changes of bone height in relation to the implants were measured using intraoral radiographs taken at insertion. The implant follow-up period ranged between $1-6$ years after implants loading. All of the implants demonstrated stability after 6 months of healing and a high implant survival rate of $98.7 \%$ was seen through the follow-up.

An average of $5.3 \pm 2.1 \mathrm{~mm}$ of intra-sinus new bone formation was observed on the radiographs. Resonance Frequency Analyses (RFM), adequate primary stability and small changes over time.

The advantages of the graftless technique as well as in other studies was presented stating that it eliminates the costs of grafting materials and reduces the risks for morbidity related to harvesting of bone grafts.

Ellegaard et al. [22] presented a study in 1997, where 24 periodontally compromised patients were treated with implant therapy of which 38 included sinus lift procedure. The patients, who where chosen for the study needed at least 2 implants each. In total, 80 implants were installed in the posterior maxilla. Fenestration was prepared in the lateral sinus wall after that the sinus membrane was lifted and the implants were inserted creating a compartment filled with blood between themselves and the sinus membrane. The implants where loaded after 5 to 6 months. During an annual follow-up, 35 out of 38 implants were successfully integrated. On a followup radiographs it was seen that new bone formation accrued around the part of the implants, which is protruding up into the sinus cavity.

A follow-up of the study above was presented in 2006 by Ellegaard et al [23]. Were tested, all patients that was treated with implant therapy between 1990 and 2002 out of 262 implants was placed in the maxillary sinus.
The results of the study had sown that similar success rate is present in implants in periodontal compromised patients as well as in ordinary implants.

\section{Development of methods improving space- maintaining management}

Recently, two studies where published, one by Johansson et al. [24] and another by Kaneko et al. [25]. Both studies already approve that sinus lifting without graft materials allows new bone formation in the sinus and in their research they use this as a fact, though both of the studies state that the amount of bone formation varies. The papers aim into improving the amount of new bone by enabling new methods for space-maintaining management. Hollow hydroxyapatite space-maintaining device was used in Johansson's et al. [24] study and titanium bone fixation device was presented in Kaneko's et al. [25] paper. Both studies concluded that predictable bone formation is attained by space-maintaining devices.

\section{DISCUSSION}

From the data that extracted from the current literature we see clear radiological evidences to bone formation in the sinus after using lateral window technique for the lift while using blood clot alone for the bone formation [15-16].

The key role to this bone formation lies in Schneiderian membrane and the bone gain does not depend much on the type of grafting material used [1]. The leading reason to the bone regeneration is the innate osteogenic potential of the Schneiderian membrane and the basic principle behind bone formation is by guided tissue regeneration.

While not all of the factors for this verity are clear, as well as the exact bone formation mechanism, it is possible that efficient space-maintaining management predicts an increase in bone gaining [24-25].

In Thor et al. [18] study it's been observed that the amount of bone gain around long implants was greater, this strengthens that the height of membrane lift is correlated with the amount of regenerated bone. Though, controversy Balleri et al. [19] study showed no such correlation.

It can be beneficial if we could identify how much bone formation we should expect since it is less invasive and cost effective procedure.

We suggest that in order to achieve better results perhaps more research must be conducted investigating the predictability of bone formation with focus on individual patient's potential for this method. 


\section{CONCLUSIONS}

It is clearly shown in the review that the potential of the maxillary sinus to heal and to form new bone without bone grafts or substitutes is of high nature. The technique presented proved to be reliable for bone augmentation of the maxillary sinus floor. As the exact mechanisms of action on how the bone formation in the sinus occurs, it is not fully cleared. More clinical studies need to be carried out to clarify the bone formation mechanism and predictability in the maxillary sinus after sinus lift without using any bone graft materials.

\section{ACKNOWLEDGMENTS AND DISCLOSURE STATEMENTS}

The authors report no conflicts of interest related to this study.

\section{REFERENCES}

1. Bell GW, Joshi BB, Macleod RI. Maxillary sinus disease: diagnosis and treatment. Br Dent J. 2011 Feb 12;210(3):113-8. [Medline: 21311531] [doi: 10.1038/sj.bdj.2011.47]

2. Tatum H Jr. Maxillary and sinus implant reconstructions. Dent Clin North Am. 1986 Apr;30(2):207-29. [Medline: $\underline{3516738]}$

3. Boyne PJ, James RA. Grafting of the maxillary sinus floor with autogenous marrow and bone. J Oral Surg. 1980 Aug;38(8):613-6. [Medline: $\underline{6993637]}$

4. Troedhan A, Kurrek A, Wainwright M. Biological Principles and Physiology of Bone Regeneration under the Schneiderian Membrane after Sinus Lift Surgery: A Radiological Study in 14 Patients Treated with the Transcrestal Hydrodynamic Ultrasonic Cavitational Sinus Lift (Intralift). Int J Dent. 2012;2012:576238. Epub 2012 Jun 17. [Medline: 22754571] [PMC free article: 3382962] [doi: 10.1155/2012/576238]

5. Moher D, Liberati A, Tetzlaff J, Altman DG; PRISMA Group. Preferred reporting items for systematic reviews and metaanalyses: the PRISMA statement. Int J Surg. 2010;8(5):336-41. Epub 2010 Feb 18. Erratum in: Int J Surg. 2010;8(8):658. [Medline: 20171303] [doi: 10.1016/j.ijsu.2010.02.007]

6. Higgins JPT, Green S. Cochrane Handbook for Systematic Reviews of Interventions. URL: http://www.cochrane.org/cochrane-interventions-handbook

7. Srouji S, Kizhner T, Ben David D, Riminucci M, Bianco P, Livne E. The Schneiderian membrane contains osteoprogenitor cells: in vivo and in vitro study.Calcif Tissue Int. 2009 Feb;84(2):138-45. Epub 2008 Dec 9. [Medline: 19067018] [doi: $10.1007 / \mathrm{s} 00223-008-9202-\mathrm{x}$ ]

8. Srouji S, Ben-David D, Lotan R, Riminucci M, Livne E, Bianco P. The innate osteogenic potential of the maxillary sinus (Schneiderian) membrane: an ectopic tissue transplant model simulating sinus lifting. Int J Oral Maxillofac Surg. 2010 Aug;39(8):793-801. Epub 2010 Apr 22. [Medline: 20417057] [doi: 10.1016/j.ijom.2010.03.009]

9. alma VC, Magro-Filho O, de Oliveria JA, Lundgren S, Salata LA, Sennerby L. Bone reformation and implant integration following maxillary sinus membrane elevation: an experimental study in primates. Clin Implant Dent Relat Res. 2006;8(1):11-24. [Medline: $\underline{16681489}$ ] [doi: $\underline{10.2310 / j .6480 .2005 .00026 . x]}$

10. Boyne PJ. Analysis of performance of root-form endosseous implants placed in the maxillary sinus. J Long Term Eff Med Implants. 1993;3(2):143-59. [Medline: 10146541]

11. Sohn DS, Moon JW, Moon KN, Cho SC, Kang PS. New bone formation in the maxillary sinus using only absorbable gelatin sponge. J Oral Maxillofac Surg. 2010 Jun;68(6):1327-33. [Medline: 20493382] [doi: 10.1016/j.joms.2010.02.014]

12. Lundgren S, Andersson S, Gualini F, Sennerby L. Bone reformation with sinus membrane elevation: a new surgical technique for maxillary sinus floor augmentation. Clin Implant Dent Relat Res. 2004;6(3):165-73. [Medline: 15726851] [doi: $10.1111 / j .1708-8208.2004 . t b 00224 . x$ ]

13. Lundgren S, Andersson S, Sennerby L. Spontaneous bone formation in themaxillary sinus after removal of a cyst: coincidence or consequence? Clin Implant Dent Relat Res. 2003;5(2):78-81. [Medline: 14536041] [doi: $10.1111 / \mathrm{j} .1708-8208.2003 . t b 00187 . x$ ]

14. Sohn DS, Lee JS, Ahn MR, Shin HI. New bone formation in the maxillary sinus without bone grafts. Implant Dent. 2008 Sep;17(3):321-31. [Medline: 18784532] [doi: 10.1097/ID.0b013e318182f01b]

15. Chen TW, Chang HS, Leung KW, Lai YL, Kao SY. Implant placement immediately after the lateral approach of the trap door window procedure to create a maxillary sinus lift without bone grafting: a 2-year retrospective evaluation of 47 implants in 33 patients. J Oral Maxillofac Surg. 2007 Nov;65(11):2324-8. [Medline: 17954333] [doi: 10.1016/j.joms.2007.06.649] 
16. Hatano N, Sennerby L, Lundgren S. Maxillary sinus augmentation using sinus membrane elevation and peripheral venous blood for implant-supported rehabilitation of the atrophic posterior maxilla: case series. Clin Implant Dent Relat Res. 2007 Sep;9(3):150-5. [Medline: 17716259] [doi: 10.1111/j.1708-8208.2007.00043.x]

17. Moon JW, Sohn DS, Heo JU, Shin HI, Jung JK. New bone formation in the maxillary sinus using peripheral venous blood alone. J Oral Maxillofac Surg. 2011 Sep;69(9):2357-67. Epub 2011 Jun 30. [Medline: 21719179] [doi: 10.1016/j.joms.2011.02.092]

18. Thor A, Sennerby L, Hirsch JM, Rasmusson L. Bone formation at the maxillary sinus floor following simultaneous elevation of the mucosal lining and implant installation without graft material: an evaluation of 20 patients treated with 44 Astra Tech implants. J Oral Maxillofac Surg. 2007 Jul;65(7 Suppl 1):64-72. Erratum in: J Oral Maxillofac Surg. 2008 Oct;66(10):2195-6. [Medline: 17586351] [doi: 10.1016/j.joms.2006.10.047]

19. Balleri P, Veltri M, Nuti N, Ferrari M. Implant placement in combination with sinus membrane elevation without biomaterials: a 1-year study on 15 patients. Clin Implant Dent Relat Res. 2012 Oct;14(5):682-9. Epub 2010 Dec 22. Erratum in: Clin Implant Dent Relat Res. 2013 May 6;15(3):470. Piero, Balleri [corrected to Balleri, Piero]; Mario, Veltri [corrected to Veltri, Mario]; Niccolò, Nuti [corrected to Nuti, Niccolò]; Marco, Ferrari [corrected to Ferrari, Marco]. [Medline: 21176096] [doi: 10.1111/j.1708-8208.2010.00318.x]

20. Lin IC, Gonzalez AM, Chang HJ, Kao SY, Chen TW. A 5-year follow-up of 80 implants in 44 patients placed immediately after the lateral trap-door window procedure to accomplish maxillary sinus elevation without bone grafting. Int J Oral Maxillofac Implants. 2011 Sep-Oct;26(5):1079-86. [Medline: 22010092]

21. Cricchio G, Sennerby L, Lundgren S. Sinus bone formation and implant survival after sinus membrane elevation and implant placement: a 1- to 6-year follow-up study. Clin Oral Implants Res. 2011 Oct;22(10):1200-12. [Medline: 21906186] [doi: 10.1111/j.1600-0501.2010.02096.x]

22. Ellegaard B, Kølsen-Petersen J, Baelum V. Implant therapy involving maxillary sinus lift in periodontally compromised patients. Clin Oral Implants Res. 1997 Aug;8(4):305-15. [Medline: 9586478] [doi: 10.1034/j.1600-0501.1997.080409.x]

23. Ellegaard B, Baelum V, Kølsen-Petersen J. Non-grafted sinus implants in periodontally compromised patients: a time-to-event analysis. Clin Oral Implants Res. 2006 Apr;17(2):156-64. [Medline: 16584411] [doi: $10.1111 / \mathrm{j} .1600-0501.2005 .01220 . \mathrm{x}]$

24. Johansson LÅ, Isaksson S, Adolfsson E, Lindh C, Sennerby L. Bone regeneration using a hollow hydroxyapatite spacemaintaining device for maxillary sinus floor augmentation--a clinical pilot study. Clin Implant Dent Relat Res. 2012 Aug;14(4):575-84. Epub 2010 Jun 2. [Medline: 20586781] [doi: 10.1111/j.1708-8208.2010.00293.x]

25. Kaneko T, Masuda I, Horie N, Shimoyama T. New bone formation in nongrafted sinus lifting with space-maintaining management: a novel technique using a titanium bone fixation device. J Oral Maxillofac Surg. 2012 Mar;70(3):e217-24. [Medline: 22374065] [doi: 10.1016/j.joms.2011.10.025]

\section{To cite this article:}

Pinchasov G, Juodzbalys G. Graft-Free Sinus Augmentation Procedure: a Literature Review.

J Oral Maxillofac Res 2014;5(1):e1

URL: http://www.ejomr.org/JOMR/archives/2014/1/e1/v5n1e1ht.pdf

doi: $10.5037 /$ jomr.2014.5101

Copyright $@$ C Pinchasov G, Juodzbalys G. Published in the JOURNAL OF ORAL \& MAXILLOFACIAL RESEARCH (http:// www.ejomr.org), 1 April 2014.

This is an open-access article, first published in the JOURNAL OF ORAL \& MAXILLOFACIAL RESEARCH, distributed under the terms of the Creative Commons Attribution-Noncommercial-No Derivative Works 3.0 Unported License, which permits unrestricted non-commercial use, distribution, and reproduction in any medium, provided the original work and is properly cited. The copyright, license information and link to the original publication on (http://www.ejomr.org) must be included. 